spirometry) were performed at baseline and 6 months after inclusion in the study. All patients were started on anti rheumatic treatment according to clinical practice with $90 \%$ of the patients started on methotrexate. In a subgroup of 15 patients bronchoscopy was performed at inclusion and 6 months later and BAL samples as well as mucosal large bronchial biopsies were retrieved. Presence of citrullinated proteins and peptydilamino deiminase (PAD) enzymes were evaluated by immunohistochemistry

Results $66 \%$ of the patients were anticitrullinated protein antibodies (ACPA) positive, $26 \%$ were current smokers and $22 \%$ have reported a previous lung pathology. All patients had active disease with a mean DAS of $5.5 \pm 1.1$ at inclusion that decreased significantly to $3.1 \pm 1.4$ after 6 months. A majority of the patients (63\%) had any type of changes on HRCT either focal or diffuse. ACPA and rheumatoid factor but not smoking status associated with the presence of changes on HRCT. Immunohistochemistry demonstrated a significant increase in expression of both PAD2 and PAD4 in the lungs of current smokers independent of the ACPA status. Results on citrullinated protein expression are pending.

Conclusion Smoking leads to high PAD expression in the lungs of RA patients already at the disease onset. However presence of ACPA, independent of smoking status, predicted the presence of lung HRCT changes. Taking together these findings suggests that smoking (and other yet unidentified factors) promotes site specific citrullination (such as in lungs) leading to generation of ACPA immunity and lung changes very early in the disease process.

\title{
A30 LUNG CHANGES ARE PRESENT IN ACPA POSITIVE RA PATIENTS ALREADY AT DISEASE ONSET
}

Gudrun Reynsdottir, Anders Harju, Marianne Engström, Sven Nyren, Magnus Sköld, Anders Eklund, Johan Grunewald, Lars Klareskog, Anca Irinel Catrina Rheumatology Department, Karolinska Institute and Karolinska University Hospital, Solna, Stockholm

\subsection{6/ard.2010.149096.30}

Objective We have previously shown that smoking increases citrullination in the lungs of healthy smokers and hypothesised that this process is an important step in the early development of rheumatoid arthritis (RA). To test this we investigated lung function and citrullination in a cohort of RA patients with very early disease, already at the time of diagnosis.

Patients and methods 108 patients, 74 female and 34 male, median age 55 (range 22-84) with symptom duration less than 1 year and time of diagnosis less than 1 week, naive to disease-modifying antirheumatic drug treatment, were included in the study and followed up for 6 months. Disease activity (DAS28) and lung function (x-ray, HRCT and dynamic 